Fecha de recepción: marzo 2020 Fecha de aceptación: abril 2020 Versión final: mayo 2020

\section{Entre el viaje y la identidad. Pertenencias. Narraciones textiles de mujeres migrantes en el Hemisferio Sur}

Marcia Veneziani ${ }^{(1)}$

\begin{abstract}
Resumen: Es un desafío para los países receptores encontrar formas, más allá de las políticas de migración implementadas- y con el fin de contribuir a la integración social- favorecer el diálogo y encuentro entre inmigrantes y sensibilizando a la población en general, en la protección de sus derechos desde una perspectiva intercultural y combatir todo tipo de violencia en las cada vez más complejas sociedades cosmopolitas. La subjetividad, tanto de los inmigrantes respecto de su nuevo entorno, como aquella de las poblaciones receptoras, resulta ser por ende, un aspecto central para aproximarse a la problemática mencionada. El factor de la identidad podría asumir, por lo tanto, una complejidad distinta, ya que conlleva el temor ancestral a la diversidad y lo extraño. A ser invadidos por ese otro. El miedo atávico a la otredad, es vivido de modo distinto según se esté de un lado o del otro del territorio en cuestión.
\end{abstract}

Palabras clave: migración - identidad- cultura - híbrido cultural - hemisferio sur - Argentina - Nueva Zelanda

[Resúmenes en inglés y portugués en la página 26]

(1) Doctora en Comunicación Social y Licenciada en Publicidad (USAL). Profesora de la Universidad de Palermo en el Área de Investigación y Producción de la Facultad de Diseño y Comunicación. Forma parte del Cuerpo Académico del Doctorado en Diseño, de la Maestría en Gestión del Diseño y del Equipo de Evaluación de Proyectos de Graduación. Es autora de los libros La Imagen de la Moda; Moda, Economía y Sociedad y de Moda, Diseño y Comunicación. Coordina publicaciones académicas internacionales con la Universidad de Palermo y la Università di Bologna (año 2013), y Parsons The New School for Design (desde el año 2012 a la actualidad), entre otras. Especialista en temas de comportamiento de Moda y Consumo. Miembro del Comité Editorial de la Revista ZoneModa Journal, dedicada a los estudios de moda en Italia, Università di Bologna, Italia, desde julio de 2013. Sus libros y ensayos académicos se encuentran incorporados como material de consulta para investigadores en el catálogo de bibliotecas de los Estados Unidos de Norteamérica y otros países de Europa y Latinoamérica. Entre otras: Library of Congress, Princeton University, Widener Library of Harvard University, Biblioteca Nacional de Suecia; Universidad Complutense de Madrid, Universidad del País Vasco, Consejo Superior de Investigaciones Científicas, Universidad de Granada, Universidad de Navarra, Red de Bibliotecas del Tecnológico de Monterrey, Universidad de Guadalajara y Universidad Autónoma de México. Fue Directora de 
las Jornadas de Moda y Pensamiento: "Hipermoda”. Fundación Ortega y Gasset Argentina (2015) Ha sido Profesora Titular en la Maestría en Comercialización y Comunicación Publicitaria en la Universidad del Salvador (USAL). A cargo de la investigación del Proyecto "Pertenencias. Narraciones Textiles de Mujeres Migrantes del Hemisferio Sur" en el MAP (Museo de Arte Popular José Hernández CABA), ganador del Premio "Maleta Abierta " del Programa Iber-Rutas junto a la Secretaría General Iberoamericana (SEGIB).

Eliseo Verón en el prólogo de la edición española del libro Antropología Estructural de Levì Strauss afirma que "los problemas de la vida social son parte de la cultura y los contenidos de ésta sirven, entre otras cosas, para definir los problemas” (1977, p. 11).

Hoy la cuestión migratoria y su impacto en la cultura, constituye una de las problemáticas a abordar de forma urgente, ya que son parte de nuestra vida social. De hecho, como se leerá más adelante en el presente escrito, tanto la OIT como la Organización de las Naciones Unidas se han pronunciado al respecto, con el fin de moderar y pautar objetivos que tienen como fin afrontar y atenuar las mismas.

El mundo actual está atravesando un cambio en variados aspectos: climáticos, económicos, políticos, flujos migratorios que se desplazan de un extremo al otro del planeta, hambrunas y retrocesos a formas de gobierno nacionalistas, conflictos armados por causas religiosas, políticas, económicas y cambios en las comunicaciones. Y las mujeres resultan ser las más vulnerables en estos procesos (Veneziani, 2020).

Estas migraciones -ya sea internacionales o interregionales- provocan en ocasiones y en las poblaciones receptoras, una serie de expresiones de resistencia (agudizadas por las crisis políticas y económicas) convirtiendo al inmigrante en el blanco de expresiones de xenofobia, racismo y discriminación.

En este sentido también estamos asistiendo a procesos a veces violentos que se manifiestan en el rechazo a personas provenientes de otras culturas y de zonas de países en conflicto y no sólo. El factor de la identidad podría asumir, por lo tanto, una complejidad distinta, ya que conlleva el temor ancestral a la diversidad y lo extraño. A ser invadidos por ese otro (Veneziani, 2012).

Es un desafío para los países receptores encontrar formas, más allá de las políticas de migración implementadas -y con el fin de contribuir a la integración social- favorecer el diálogo y encuentro entre inmigrantes y sensibilizando a la población en general, en la protección de sus derechos desde una perspectiva intercultural y combatir todo tipo de violencia en las cada vez más complejas sociedades cosmopolitas.

Durante la última década, se advierte un cambio en los flujos migratorios más allá de la ruta Sur - Norte (aquí se hace referencia como punto de origen a países latinoamericanos) hacia Europa y EEUU), un aumento de aquellos hacia Oceanía (Australia y Nueva Zelanda) como así también, entre los mismos países del sur, en búsqueda de nuevos horizontes laborales y personales. 
Según la Organización Internacional del Trabajo (OIT) (2017) se observa una tendencia reveladora: "el aumento de la migración dentro y entre las regiones del hemisferio sur. En la actualidad, la mayoría de los trabajadores migrantes (51,5 por ciento) vive en los países del Sur".

La actual población de la Argentina es de casi 44 millones de habitantes, y una bajísima tasa de densidad de habitantes, de 16 por kilómetro cuadrado. Con alta concentración en lo que se llama el Gran Buenos Aires (cerca de 30\% el total); casi totalmente urbana (algo más de 92\%) Argentina llegará a su pico poblacional en 2065, cuando alcance los 51.377.000 de habitantes, según el informe «Perspectivas de la población mundial», realizado por el Departamento de Asuntos Económicos y Sociales de la ONU. A partir de ahí, comenzará a descender hasta situarse en 49 millones para 2100. Eso siempre que el efecto de migraciones importantes y llegada de refugiados, no altere el pronóstico (Gómez, 2018 s/p).

Otro detalle del informe de la OIT (2017) remarca "la creciente feminización de la migración laboral" a nivel global. El 44 por ciento de todos los migrantes son mujeres y entre los trabajadores domésticos, la proporción de aquellas supera el 73 por ciento.

Tomando como referencia otros estudios académicos previos relacionados con la situación de las mujeres migrantes se indica que:

Una vez en destino, la inserción de las mujeres migrantes en los mercados de trabajo de los países industriales avanzados ocurre en términos de segmentación, fenómeno que se comporta de manera muy similar en los países en vías de desarrollo. Este proceso se traduce en que para la gran mayoría de inmigrantes las oportunidades laborales se presentan sólo en aquellos empleos menos deseados por los nacionales, entre otros motivos porque la remuneración suele ser más baja que en el resto, tienen mayores niveles de precariedad laboral y son trabajos más devaluados socialmente. En consecuencia, son las mujeres las que terminan ocupando los empleos de menor prestigio (Acosta González, 2013, s/p).

También Cerrutti (año 2018) sostiene que en los grupos en los que no prevalece el trabajo vinculado al servicio doméstico o personal, "el balance entre sexos es más equilibrado. Esto sucede con la migración boliviana, que se caracteriza por una fuerte centralidad en las actividades económicas de tipo familiar, como la horticultura, el comercio informal, la venta ambulante o los talleres textiles" (en Gómez, $2018 \mathrm{~s} / \mathrm{p}$ ).

El ensayo que aquí nos convoca, hace referencia a mujeres migrantes que se desplazan específicamente dentro del hemisferio austral (internacionales, interregionales o dentro de sus mismos países) -especialmente desde el ámbito rural al urbano-y una reflexión acerca de su repercusión en las culturas receptoras.

La subjetividad, tanto de los inmigrantes respecto de su nuevo entorno, como aquella de las poblaciones receptoras, resulta ser por ende, un aspecto central para aproximarse a la 
problemática mencionada. Así, se plantean una serie de interrogantes que ameritan más que una simple cavilación al respecto: ¿Cómo es la vivencia de aquellas que llegan y de quienes las reciben? ¿Cómo son percibidas estas identidades ajenas a la propia por ambas partes y las posibles prácticas que se consiguen producir para lograr su integración? ¿Cómo estas nuevas identidades pueden entrelazarse y generar híbridos culturales que promuevan el bienestar del inmigrante?

El proyecto Pertenencias, narraciones textiles de mujeres migrantes en el hemisferio sur, realizado en el Museo de Arte Popular José Hernández (MAP- GCBA) en el año 2019 apuntó, a visibilizar la problemática de las mujeres migrantes en dos países (Argentina y Nueva Zelanda).

El mismo comprendió tres aspectos:

1. Una exposición de los trabajos realizados por mujeres desplazadas en ambos países.

2. Talleres de intercambio de saberes entre las participantes.

3. Coloquios de referentes de la problemática abordada, ONG, académicos y líderes de las comunidades de los dos países.

En el caso específico de la Argentina, la intención fue, además, ofrecer alternativas viables de inserción social y laboral a mujeres de bajos recursos que se encuentran inmersas en realidades complejas por haber migrado y cambiado su modo de vida del ámbito rural, también pobre pero conectado con la naturaleza, a otro urbano.

Las mujeres inmigrantes se suelen asentar en la periferia de las grandes ciudades, donde transitan diariamente conflictos relacionados con la pobreza, la desocupación y la violencia. Ellas y sus familias se encuentran expuestas a la delincuencia, la droga, el clientelismo político y la falta de perspectiva a futuro.

En el caso de Nueva Zelanda, Victoria Martínez Azaro presentó The Travelling Sewing Box Project, su proyecto personal que comenzó en el año 2017 asistiendo a mujeres refugiadas e inmigrantes latinoamericanas en su integración social y cultural, por medio de la exploración del arte textil. La extensa tela que se expuso en Buenos Aires, fue el resultado del trabajo colectivo que plasmó las historias de las migrantes sudamericanas en el país Oceánico. El mismo cuenta con el auspicio de ALAC (Aotearoa Latin American Community Incorporated), la asociación que colabora con el Ministerio de Desarrollo Social de ese país en la integración cultural y económica de los inmigrantes latinos.

$\mathrm{Al}$ mismo tiempo, Pertenencias, narraciones textiles de mujeres migrantes en el hemisferio sur, asentado para la ocasión en el Museo de Arte Popular José Hernández (MAP- GCBA) de la ciudad de Buenos Aires, Argentina, desarrollado por quien escribe el presente ensayo y tiene a cargo la investigación (Marcia Veneziani) y su curadora Ximena Eliçabe, pretendió poner en evidencia y colaborar en la preservación de las raíces culturales, los vínculos familiares y étnicos de las comunidades a las que pertenecen las mujeres migrantes que participaron de la actividad.

La mayoría de ellas son originarias de las provincias de Catamarca, Entre Ríos y del país vecino, Paraguay, poseían conocimientos previos de tejido en telar, ñandutí (que significa "tela de araña" en guaraní) y de tejido a dos agujas. Continúan con la actividad que aprendieron de niñas y en su entorno familiar. 
Se desarrollaron actividades que pretendieron reflexionar cómo dignificar su rol en la sociedad, evitar el desarraigo con el fin de promover su inclusión y diversidad.

Para ello, la investigación y posterior puesta en marcha resultó esencial.

La pertinencia del proyecto mencionado y seleccionado entre los ganadores del Fondo Maleta Abierta, Programa IBER-RUTAS (Fortalecimiento de rutas de derechos e interculturalidad en la migración iberoamericana) de la Secretaría General Iberoamericana, se alinea con los objetivos propuestos por el Pacto Mundial de la Inmigración auspiciado por las Naciones Unidas en el año 2018.

Recordemos que luego de más de un año y medio de consultas y negociaciones (iniciadas en al año 2017) el 13 de julio de 2018, los Estados miembros de las Naciones Unidas finalizaron el texto del Pacto Global para la migración segura, ordenada y regular.

Para afrontar estos nuevos desafíos, se estableció un primer acuerdo entre gobiernos con el objeto de "cubrir todas las dimensiones de la migración internacional de una manera integral y completa" (Global Compact for Migration, 2018).

En el mismo, se establecieron 23 objetivos con el fin de abordar la cuestión migratoria examinando todos los niveles (local, nacional, regional y global) y cuyo propósito sería:

- mitigar los factores adversos y los factores estructurales que impiden a las personas construir y mantener medios de vida sostenibles en sus países de origen; - reducir los riesgos y las vulnerabilidades que enfrentan los migrantes en diferentes etapas de la migración al respetar, proteger y cumplir sus derechos humanos y brindarles atención y asistencia;

- abordar las preocupaciones legítimas de los Estados y las comunidades, al tiempo que reconoce que las sociedades están experimentando cambios demográficos, económicos, sociales y ambientales en diferentes escalas que pueden tener implicaciones y resultar de la migración;

- crear condiciones propicias que permitan a todos los migrantes enriquecer nuestras sociedades a través de sus capacidades humanas, económicas y sociales, y así facilitar sus contribuciones al desarrollo sostenible a nivel local, nacional, regional y global (Global Compact for Migration, 2018).

\section{¿Quiénes son ellos? La otredad como estado anímico del migrante}

-¡Ah!, le dije, esta mañana he llegado pasando por tristes lugares, y estoy aún en la primera vida; aunque al hacer este viaje, voy preparándome para la otra. La Divina Comedia

El Purgatorio, CANTO VIII (1945, p. 201) 
En la actualidad, la migración impacta directamente en el modo en que el sujeto involucrado en la misma, vivencia la propia identidad y no sólo. Se idealiza tanto el territorio de partida como el de recepción.

Las nuevas tecnologías permiten acceder a otras culturas y territorios antes impensados. En el propio suelo y por medio de una pantalla, se puede informar de otros lugares que prometen bienestar y alivio para atenuar las carencias del sitio de origen.

El inmigrante se debate entre la pertenencia, la alteridad y la transformación de sus relaciones sociales y afectivas -tanto dentro como fuera de ambos espacios- que se producen como consecuencia de la búsqueda de un bienestar económico.

"Uno puede incluso empezar a sentirse en cualquier parte chez soi, 'en casa', pero hay que pagar el precio de aceptar que no se estará verdadera y totalmente en casa en ninguna parte" (Bauman, 2007, p. 36).

Y es en esos limbos anímicos (no ser ni de un lado ni del otro) que al modo de los no lugares -que tan claramente describió Marc Augé como los nuevos espacios de nuestra modernidad- es donde se debate el espíritu del migrante: “...también los campos de tránsito prolongado donde se estacionan los refugiados del planeta” (2000, p. 41).

La emergencia y el crecimiento de identidades y prácticas transnacionales han venido a poner en evidencia la novedad y complejidad del hecho social que están produciendo las migraciones contemporáneas. Muchos y cada vez más migrantes construyen sus trayectorias como una experiencia que conecta en un solo espacio los lugares de origen y procedencia. Estos migrantes son sujetos que no dejan completamente de partir del lugar de procedencia, pero que tampoco acaban nunca de llegar al de destino. Están material y simbólicamente situados aquí y allá, construyendo un proyecto de vida en un espacio que trasciende la inmediatez local, y que sin embargo, se construye cotidianamente en esa inmediatez dual (Elizalde ed alt., $2013 \mathrm{~s} / \mathrm{p}$ ).

Las emergencias políticas y económicas de los territorios natales obligan a las personas migrantes a dejar no solo la propia tierra, sino también su cultura y lazos afectivos.

La sensación de vacuidad interior que se puede percibir al llegar a la tierra prometida va más allá de la adquisición de un cierto bienestar económico.

El amparo ante este traspaso puede temperarse mediante la pertenencia a grupos integrados por otros migrantes y locales. “(...) intimidad y distancia crean una situación privilegiada. Ambas son necesarias” (Juan Goytisolo en Bauman, 2007, p. 37).

Es decir, el pertenecer a un colectivo menguaría la sensación de angustia de autopercepción de aquella diversidad cultural.

Nuestra época se nos presenta entonces como paradójica, ya que mientras "la unidad del espacio terrestre se vuelve pensable y en el que se refuerzan las grandes redes multinacionales, se amplifica el clamor de los particularismos" (Augé, 2000, p. 41).

Es pues, en las tierras receptoras donde el migrante choca, además de la realidad de las leyes migratorias, con factores culturales que desencadenan grandes polémicas y donde se percibe la singularidad de aquellos que pretenden permanecer aislados o de quienes desean acompañar los procesos actuales de integración en las modernas sociedades mul- 
ticulturales. Es decir, el primer caso puede llevar a la adhesión, por parte de los locales, a nostálgicas ideologías nacionalistas, como modo de defender la propia identidad o el del intercambio de un futuro posible que incluya al otro y su cultura.

"Como si el conservadurismo de los unos y el mesianismo de los otros estuviesen condenados a hablar el mismo lenguaje: el de la tierra y el de las raíces” (Augé 2000, p. 41).

La cuestión identitaria concierne necesariamente al otro, y depende además, del contexto en el cual se desarrolla ese vínculo. Es por esa misma razón, que la identidad se encuentra en estado de evolución permanente. Allí, se desarrolla el mecanismo entre el forastero y el lugareño que a su vez, también va cambiando. Obedece a esos contextos y a cómo se desarrolle ese vínculo entre ambos, la gestación de posibles híbridos culturales capaces de evidenciar esos espacios inciertos: "Una zona de frontera es un lugar vago e indeterminado creado por el residuo emocional de un límite artificial (...) Las personas que habitan las dos realidades están obligadas a vivir en la interfaz de ambas" (Anzaldúa 1987, en Grossberg, 2011, p. 156).

Tal como se ha analizado en estudios previos (Veneziani, 2012) también estamos asistiendo a procesos a veces violentos que se manifiestan en el rechazo a personas provenientes de otras culturas y de zonas de países en conflicto.

El factor de la identidad podría asumir, por lo tanto, una complejidad distinta, ya que conlleva el temor ancestral a la diversidad y lo extraño. A ser invadidos por ese otro.

Ese miedo atávico a la otredad, es vivido de modo distinto según se esté de un lado o del otro del territorio en cuestión.

Para Bauman (2007) el inmigrante se ve siempre obligado a explicar, negociar, poner en evidencia u ocultar. "Existen diferencias que limar o que paliar o que, por el contrario, hay que agudizar más y hacer más legibles. Aquí las 'identidades' flotan en el aire, algunas elegidas por uno pero otras infladas y lanzadas por quienes nos rodean” (p. 35).

Umberto Eco (1998) distingue los conceptos de migración e inmigración: mientras esta última es controlada políticamente, a la primera la compara con los fenómenos naturales, ya que la considera incontrolable: "cuando todo un pueblo, poco a poco, se desplaza de un territorio a otro ( $\mathrm{y}$ no tiene importancia cuántos permanecen en el territorio original, sino en qué medida los migrantes cambian radicalmente la cultura del territorio al que han migrado)" (p. 122).

Independientemente de la categoría adjudicada por el estudioso italiano, lo cierto es que la cultura de los migrantes o inmigrantes impacta seguramente en la tierra receptora y viceversa, lo que puede llevar a choques culturales que es necesario moderar.

El mundo ha cambiado. Las economías de mercado, el avance de las nuevas tecnologías y la globalización han transformado nuestras modernas sociedades en ciudades cosmopolitas donde conviven personas provenientes de todo el planeta: se hablan pluralidad de lenguas, se comen platos provenientes de diversas culturas, se escuchan infinidad de melodías y canciones, se forman familias cuyos integrantes provienen de las más remotas partes del mundo.

Por más murallas que se construyan para impedir su avance, el desarrollo tecnológico y las comunicaciones nos indican el camino hacia un nuevo modelo de convivencia.

Ya no es todo sí o no, ocurre o no ocurre, se comienzan a considerar las probabilidades de ocurrencia. La naturaleza se comporta de manera muy compleja (y por ende, el ser 
humano como parte de ésta, también). En las comunicaciones luchamos siempre contra la entropía (segunda ley de la termodinámica que concluye que la naturaleza tiende al desorden), y a la misma tendencia de la entropía a aumentar, como lo demostró Gibbs y nos lo recuerda Wiener (quien trató de explicar la aplicación de las leyes físicas al mundo de la comunicación):

En el universo de Gibbs el orden es menos probable, el caos más probable. Pero mientras el universo en su totalidad, si existe en cuanto tal, tiende a ese estado definitivo, existen enclavados locales, cuya dirección parece opuesta a la del universo como un todo en los cuales hay una tendencia temporal y limitada a aumentar la complejidad de su organización. La vida encuentra asilo en algunos de esos enclavados (Wiener, 1969, p. 14).

\section{Conclusión}

No hay progreso sin investigación. Esta resultó cardinal a la hora de emprender el proyecto. Mi viaje de exploración a Nueva Zelanda en el año 2018 representó una pieza esencial para poner el foco en la problemática abordada en el presente trabajo.

Para la puesta en marcha del mismo fue necesario sondear en parajes desconocidos y funcionar luego, como eslabón entre investigadores de ambos países para continuar elaborando futuros estudios.

La memoria de las migrantes y refugiadas del hemisferio sur se constituyó en el eje central de la investigación del proyecto.

Así, por medio del diálogo entre miembros de las distintas comunidades se pretendió, no solo poner en común experiencias de mujeres migrantes, sino además, generar sinergia al intercambiar técnicas textiles y artesanales que permitieron enriquecer la puesta en valor de la historia y experiencias personales de quienes conviven en un mismo territorio. En este caso específico, el de dos países: Argentina y Nueva Zelanda.

Se pudo verificar que más allá de las circunstancias políticas, económicas, geográficas y culturales, por medio del lazo que se establece entre ambas partes, en este caso, mediante el diseño de piezas comunitarias, se pueden generar espacios de creación posibles para amainar las rispideces que surgen durante la fase de integración; ya que: "las identidades son siempre relacionales e incompletas, siempre están en proceso. Toda identidad depende de su diferencia y su negación de algún otro término, mientras que la identidad de este depende de su diferencia y su negación de la primera" (Grossberg en Hall, y du Gay, 2011, p. 152).

Por ende, el factor de la compatibilidad en el Proyecto Pertenencias. Narraciones textiles de mujeres en el hemisferio sur que incluyó el trabajo de Victoria Martínez Azaro: The Travelling Sewing Box Project auspiciado por del ALAC (Aotearoa Latin American Community Incorporate) se torna paradigmático como experiencia para continuar ahondando en otros caminos posibles que contribuyan a la integración social de migrantes y refugiados en los países de acogida. 
Es decir, la afirmación de la propia identidad plasmada (en este caso específico) en artesanías o textiles pone en valor, no solo la historia de las propias comunidades en los países de recepción, sino también contribuye a la creación de híbridos culturales que favorezcan la unificación de ambas partes.

Las historias de vida de los seres humanos están íntimamente ligadas a las historias de vida de los objetos: se encuentran, se tejen y se entrelazan; no es posible narrar la historia de una persona o de un grupo sin narrar la historia de vida de los objetos con los cuales interactúa de manera constante, y viceversa. De igual modo, es posible comprender la historia de una comunidad a través de la historia de vida de los objetos que los rodean, que usan o producen (Martínez González, 2017, p. 328).

\section{Bibliografía}

Acosta González, E. "Mujeres migrantes cuidadoras en flujos migratorios sur-sur y sur-norte: expectativas, experiencias y valoraciones", Polis. $\mathrm{N}^{\circ} 35,13$ octubre 2013, Recuperado de: http://journals.openedition.org/polis/9247

Alighieri, D. (1945). La Divina Comedia, Buenos Aires: Anaconda.

Augé, M. (2000). Los no lugares. Espacios del anonimato. Una antropología de la sobremodernidad. Barcelona: Gedisa.

Bauman, Z. (2016). Extraños llamando a la puerta. Buenos Aires: Paidós.

Bauman, Z. (2017). Identidad, Buenos Aires: Losada.

Cerrutti, M. La Argentina en el Siglo XXI en Gómez, B. no. 1205 (jun. 2018) "Migración y refugiados. El desafío demográfico". Mercado. Buenos Aires.

Eco, U. (1998). Cinco escritos morales, Barcelona: Lumen.

Elizalde, A.; Thayer Correa, L. y Córdova, M. (2013). "Migraciones sur-sur: paradojas globales y promesas locales".Polis $\mathrm{N}^{\circ} 35,15$ octubre 2013. Recuperado de URL: https:// journals.openedition.org/polis/9375http://journals.openedition.org/polis/9375

Gómez, B. no. 1205 (jun. 2018) “Migración y refugiados. El desafío demográfico”. Mercado. Buenos Aires.

Grossberg, L. (2011). “Identidad y estudios culturales: ¿no hay nada más que eso?”. Cuestiones de identidad cultural, Hall, S y du Gay, P., Buenos Aires: Amorrortu.

Lévi -Strauss, C. [1958] (1977). Antropología Estructural, Buenos Aires: Eudeba.

Martínez González, M (2017). Tejiendo destinos. La antropología y el diseño en el estudio de los objetos de palma. México: Universidad Nacional Autónoma de México.

OIT (2 de junio de 2017). "La Conferencia Internacional del Trabajo discutirá sobre la migración laboral y la contratación equitativa”. Recuperado de: http://www.ilo.org/global/ about-the-ilo/newsroom/news/WCMS_556327/lang--es/index.htm

Organización de las Naciones Unidas (2018). Refugiados y Migrantes. "Global Compact for Migration, 2018” Recuperado de: https://refugeesmigrants.un.org/migration-compact 
Organización Internacional para las migraciones (2019). La Migración en América del Sur Tendencias y datos relevantes. Recuperado de: https://robuenosaires.iom.int/tendenciasy-datos-relevantes

Veneziani, M. (2012). “La impronta de la identidad en un mundo globalizado". Actas de Diseño No 13 Año VI, Julio 2012. Buenos Aires.: Universidad de Palermo, Facultad de Diseño y Comunicación.

Veneziani, M. (2020). "Prólogo". Tiempos inestables. Un mundo en transición, Cuadernos del Centro de Estudios en Diseño y Comunicación [Ensayos] Número 78. Año 20. Marzo de 2020. Buenos Aires: Universidad de Palermo, Facultad de Diseño y Comunicación. Wiener N. (1969). Cibernética y Sociedad, Buenos Aires: Sudamericana.

\begin{abstract}
It presents a challenge for the host countries to find ways, beyond the implementation of various migration policies, to encourage dialogue and encounter between immigrants by creating awareness among the population at large, in order to contribute to social integration, and to protect their rights from a cross-cultural perspective, to fight against all kinds of violence that arise in the increasingly complex cosmopolitan societies. Subectivity -both the immigrant's one regarding their new environment, and that of the host populations- turns out to be, therefore, a key aspect in the approach of the aforementioned problem. The identity factor could, therefore, add a different complexity, since it entails the ancestral fear of diversity and what is strange. To be "invaded" by that "other", the atavistic fear of otherness, is felt differently depending on whether one is on one side or the other of the territory in question.
\end{abstract}

Keywords: migration - cultural identity - culture - cultural hybrid - Souther Hemisphere - Argentina - New Zealand.

Resumo: Para os países receptores é um desafio encontrar formas para além das políticas de migração implementadas -e com a finalidade de contribuir para a integração social-, favorecer o diálogo e o encontro entre imigrantes sensibilizando à população em geral no tema da proteção dos seus direitos a partir de uma perspectiva intercultural, assim como combater todo tipo de violência nas cada vez mais complexas sociedades cosmopolitas. A subjetividade, tanto dos imigrantes a respeito do seu novo entorno, quanto a das populações receptoras resulta, portanto, numa complexidade diferente, visto que acarreta o temor ancestral à diversidade e ao estranho. A serem invadidos por esse outro. Este medo atávico à alteridade é vivido de forma diferente conforme o sujeito esteja de um lado ou de outro do território em pauta.

Palavras chave: migração - identidade- cultura - hibridismo cultural - hemisfério sul Argentina - Nova Zelândia.

[Las traducciones de los abstracts fueron supervisadas por el autor de cada artículo] 